\title{
CONTRIBUTION OF THE ELECTRIC DOUBLE LAYER TO THE CURVATURE ELASTICITY OF CHARGED AMPHIPHILIC MONOLAYERS
}

\author{
H.N.W. LEKKERKERKER \\ Van 't Hoff Laboratory, University of Utrecht, Padualaan 8, 3584 CH Utrecht, \\ The Netherlands
}

Received 6 June 1989

The electrostatic contribution to the bending moduli of an amphiphilic monolayer is calculated on the basis of the Poisson-Boltzmann equation. The electrical free energy for a spherical and cylindrical surface is expanded in inverse powers of the radius of curvature $a$. The coefficient $(1 / a)^{2}$ in the electrical free energy gives the bending elastic moduli.

\section{Introduction}

In recent years it has become increasingly clear that for the understanding of the physics of amphiphilic monolayers and bilayers the curvature elastic properties $[1,2]$ of these systems play a key role [3]. For example the intricate phase behaviour of microemuisions appears to be largely controlled by the curvature elastic moduli of the amphiphilic layers in these systems $[4,5]$. Also the various equilibrium configurations of fluid membranes are determined by their curvature elasticity [6].

Measurements based on the analysis of thermally excited shape fluctuations [7-11] and micromechanical measurements [12] yield for the bending elastic modulus $K$ of simple lipid bilayers a value of $(1-2) \times 10^{-19} \mathrm{~J}$, i.e. on the order of tens of $k_{\mathrm{B}} T$. This value agrees remarkably with an early estimate [1] based on the curvature elasticity of liquid crystals. On the other hand for the bending elastic modulus of the amphiphilic layers in microemulsion systems one finds with various techniques (ESR [13], dynamic light scattering $[14,15]$, ellipsometry $[16,17], \mathrm{X}$-ray scattering $[18,19])$ a value of $(0.2-2) k_{\mathrm{B}} T$, i.e. typically one order of magnitude :ower than in the case of lipid bilayers. The high flexibility of the amphiphilic layers in microemulsions has been invoked to explain the variety of phases in these systems $[4,5]$.

0378-4371/89/\$03.50 C Elsevier Science Publishers B.V.

(North-Holland Physics Publishing Division) 
The small value of the bending elastic modulus $K$ in microemulsion systems appears to be intimately connected with the fact that the amphiphilic layers in these systems are composed of a surfactant and a shorter chain cosurfactant (alcohol). Recently Szleifer et al. [20] presented a statistical thermodynamic theory to explain the decrease of the bending elastic modulus upon replacement of a fraction of the long chains by shorter ones. These authors pursued the premise that the curvature elasticity of surfactant films is dominated by the "tails" - rather than by the "heads" - of the constituent amphiphiles. However electrostatic energies do play a role in microemulsions and also in membrane systems. They contribute to the interfacial tension [21] and to the interfacial bending stress [22]. Recently Winterhalter and Helfrich [23] calculated the electrostatic contribution to the curvature elastic moduli using the linearized Poisson-Boltzmann equation (Debye-Hücket approximation). This approximation is only valid for low potentials, i.e. low surface charges. Here we show how the electrostatic contribution to the curvature elastic parameters can be calculated on the basis of the non-linear Poisson-Boltzmann equation. Starting from this equation the electrical free energy of a charged spherical and cylindrical is obtained as a series in inverse powers of the radius of curvature $a$. From the coefficient of the $(1 / a)^{2}$ term in the electrical free energy the electrostatic contribution to the curvature elastic moduli is obtained.

The problem treated here bears a close relation to the problem of the determination of the electrostatic contribution to the stiffness of wormlike polyelectrolytes. The bending elastic modulus of polyelectrolytes has an intrinsic part (the bending elastic modulus of the corresponding uncharged polymer) and an electric part. The electrostatic contribution was first determined by Odijk [24] and independently by Skolnick and Fixman [25] in the DebyeHückel approximation. Later Le Bret [26] and Fixman [27] calculated the electrostatic contribution to the bending elastic modulus using the non-linear Poisson-Boltzmann equation. In fact the determination of the electrostatic contribution to the curvature elastic parameters of layers turns out to be simpler than the corresponding calculation of the electrostatic contribution to the bending modulus of polyelectrolytes. Whereas in the latter case one has to resort to numerical calculations to obtain resuits, in the case of layers analytic expressions can be obtained for the electrostatic contribution to the curvature elastic moduli.

2. Calculation of the electrical free energy of a charged spherical and cylindrical surface

The electrical free energy per unit area of the double layer is given by [28] 


$$
f_{\mathrm{el}}=\int_{0}^{\sigma} \psi_{0}\left(\sigma^{\prime}\right) \mathrm{d} \sigma^{\prime} .
$$

Here $\sigma$ is the surface charge density and $\psi_{0}$ is the potential at the surface. Finding the relation between the surface charge density and the surface potential for a charged spherical or cylindrical surface is difficult, because this involves solutions of the Poisson-Boltzmann equation for spherical or cylindrical symmetry for which no closed solutions exist. Extending the work of Dukhin et al. [29] and Chew and Sen [30] we obtain for the case of thin double layers ( $\kappa a \gg 1, a$ radius of the particle, $\kappa$ inverse Debye length) a series expansion in $1 / \kappa a$ for $\psi_{0}$ up to terms of order $1 /(\kappa a)^{2}$. Using these results we are able to calculate the electrical free energy up to terms of order of $1 /(\kappa a)^{2}$ and from there on we obtain the electrical contribution to the curvature elasticity.

\subsection{Charged spherical surface}

The Poisson-Boltzmann equation for a charged spherical surface immersed in a $:-1$ electrolyte can be written as

$$
\frac{\mathrm{d}^{2} \Psi}{\mathrm{d} r^{2}}+\frac{2}{r} \frac{\mathrm{d} \Psi}{\mathrm{d} r}=\kappa^{2} \sinh \Psi
$$

Here $r$ is the radial coordinate, $\Psi$ is the dimensionless potential,

$$
\Psi=\frac{e \psi}{k_{\mathrm{B}} T}
$$

and $\kappa$ is the inverse Debye length,

$$
\kappa=\left(\frac{2 e^{2} n_{\mathrm{dl}}}{\varepsilon_{0} \varepsilon_{\mathrm{r}} k_{\mathrm{B}} T}\right)^{1 / 2}
$$

where $e$ is the elementary charge, $k_{\mathrm{B}}$ is Boltzmann's constant, $T$ is the absolute temperature, $n_{\mathrm{el}}$ is the number of molecules of electrolyte per unit volume, $\varepsilon_{\mathrm{r}}$ is the dielectric constant of the aqueous medium and $\varepsilon_{0}$ is the permittivity of the vacuum.

In fact a full solution of the Poisson-Boltzmann equation (2) is not needed. For the calculation of the electrical free energy (eq. (1)) it is only necessary to know how the potential at the surface varies with the surface charge density. Therefore we consider the solution of eq. (2) near the surface. We consider the case of a double layer outside a sphere. Taking $1 / \kappa a$ as the small parameter we 
write

$$
\Psi=\Psi^{(0)}+\frac{1}{\kappa a} \Psi^{(1)}+\frac{1}{(\kappa a)^{2}} \Psi^{(2)}+\ldots
$$

Substituting eq. (5) in eq. (2) and making the coordinate transformation

$$
r=a+x / \kappa
$$

we find

$$
\begin{aligned}
& \frac{\mathrm{d}}{\mathrm{d} x^{2}}\left[\Psi^{(0)}+\frac{1}{\kappa a} \Psi^{(1)}+\ldots\right]+\frac{2 / \kappa a}{1+x / \kappa a} \frac{\mathrm{d}}{\mathrm{d} x}\left[\Psi^{(0)}+\frac{1}{\kappa a} \Psi^{(1)}+\ldots\right] \\
& \quad=\sinh \left[\Psi^{(0)}+\frac{1}{\kappa a} \Psi^{(1)}+\ldots\right] .
\end{aligned}
$$

We now assume that $x$ is sufficiently small such that

$$
\frac{x}{\kappa a} \ll 1
$$

and thus we may write

$$
\frac{1}{1+x / a \kappa} \cong 1-\frac{x}{\kappa a}+\ldots
$$

Using this expansion and equating terms of the same power of $1 / \kappa a$ on both sides of eq. (7) gives the following equations:

$$
\begin{aligned}
& \frac{\mathrm{d}^{2} \Psi^{(1)}}{\mathrm{d} x^{2}}=\sinh \Psi^{(0)}, \\
& \frac{\mathrm{d}^{2}}{\mathrm{~d} x^{2}} \Psi^{(1)}-\Psi^{(1)} \cosh \left(\Psi^{(0)}\right)=-2 \frac{\mathrm{d}}{\mathrm{d} x} \Psi^{(0)}, \\
& \frac{\mathrm{d}^{2}}{\mathrm{~d} x^{2}} \Psi^{(2)}-\Psi^{(2)} \cosh \left(\Psi^{(0)}\right)=-2 \frac{\mathrm{d}}{\mathrm{d} x} \Psi^{(1)}+2 x \frac{\mathrm{d}}{\mathrm{d} x} \Psi^{(1)} \\
&+\frac{1}{2}\left(\Psi^{(1)}\right)^{2} \sinh \left(\Psi^{(0)}\right) .
\end{aligned}
$$

With the boundary condition that

$$
\Psi^{(1)}=\Psi^{(1)}=\Psi^{(2)}=\ldots=0 \quad \text { as } x \rightarrow x
$$


one obtains from eqs. (8)-(10)

$$
\begin{aligned}
& \frac{\mathrm{d} \Psi^{(0)}}{\mathrm{d} x}=-2 \sinh \left(\frac{1}{2} \Psi^{(0)}\right) \\
& \frac{\mathrm{d} \Psi^{(1)}}{\mathrm{d} x}+\Psi^{(1)} \cosh \left(\frac{1}{2} \Psi^{(0)}\right)=-4 \tanh \left(\frac{1}{4} \Psi^{(0)}\right) \\
& \frac{\mathrm{d} \Psi^{(2)}}{\mathrm{d} x}+\Psi^{(2)} \cosh \left(\frac{1}{2} \Psi^{(0)}\right)=-\frac{1}{4}\left(\Psi^{(1)}\right)^{2} \sinh \left(\frac{1}{2} \Psi^{(0)}\right)-\frac{2 \Psi^{(1)}}{\cosh \left(\frac{1}{2} \Psi^{(0)}\right)+1} \\
& \quad+4 x \tanh \left(\frac{1}{4} \Psi^{(0)}\right)+\frac{4\left[\tanh ^{2}\left(\frac{1}{4} \Psi^{(0)}\right)-2 \ln \cosh \left(\frac{1}{4} \Psi^{(0)}\right)\right]}{\sinh \left(\frac{1}{2} \Psi^{(0)}\right)}
\end{aligned}
$$

Assuming that we are dealing with a constant surface charge the boundary conditicns at the surface are

$$
\left(\frac{\mathrm{d} \Psi^{(0)}}{\mathrm{d} x}\right)_{x=0}=\left(\frac{\mathrm{d} \Psi}{\mathrm{d} x}\right)_{x=0}, \quad\left(\frac{\mathrm{d} \Psi^{(1)}}{\mathrm{d} x}\right)_{x=0}=\left(\frac{\mathrm{d} \Psi^{(2)}}{\mathrm{d} x}\right)_{x=0}=\ldots=0 .
$$

Using these boundary condiiions one obtains from eqs. (12)-(14) to order $1 /(\kappa a)^{2}$ the following expression for the surface potential:

$$
\begin{aligned}
\Psi_{0}= & 2 \ln (p+q)-\frac{1}{\kappa a} \frac{4(q-1)}{p q} \\
& +\frac{1}{(\kappa a)^{2}}\left\{\frac{4(q-1)^{2}(2 q+1)}{p^{3} q^{3}}-\frac{4 \ln \left(\frac{1}{2}[q+1]\right)}{p q}\right\},
\end{aligned}
$$

where

$$
p=\frac{1}{2}\left|\left(\frac{\mathrm{d} \Psi}{\mathrm{d} x}\right)_{x=0}\right|
$$

and

$$
q=\sqrt{p^{2}+1}
$$

(In writing down eq. (17) we have assumed that we are dealing with a situation where $\Psi_{0}$ is positive.) Through the equation of Gauss the quantity $p$ is related to the surface charge density $\sigma$,

$$
p=\frac{2 \pi Q|\sigma|}{\kappa e},
$$


where $Q$ is the Bjerrum length,

$$
Q=\frac{e^{2}}{4 \pi \varepsilon_{0} \varepsilon_{\mathrm{r}} k_{\mathrm{B}} T}
$$

$(Q=0.714 \mathrm{~nm}$ for aqueous solutions at $298 \mathrm{~K})$.

Hence it follows that eq. (17) represents a relation between the surface potential and the surface charge density. Now the integral in the expression (1) for the electrical free energy can be calculated, resulting in

$$
\begin{aligned}
f_{\mathrm{el}}^{\text {sphere }}= & \left(\frac{k_{\mathrm{B}} T}{e}\right)^{2} \varepsilon_{\mathrm{r}} \varepsilon_{0} \kappa\left[\{4(p \ln (p+q)-q+1)\}-\frac{8}{\kappa a} \ln \left(\frac{1}{2}[q+1]\right)\right. \\
& \left.+\frac{4}{(\kappa a)^{2}}\left\{\frac{(q-1)(q+2)}{(q+1) q}-\int_{z=2 /(1+q)}^{z=1} \frac{\ln z}{z-1} \mathrm{~d} z\right\}\right] .
\end{aligned}
$$

A similar result was first obtained by Stokes [31] using a slightly different method.

\subsection{Charged cylindrical surface}

The Poisson-Boltzmann equation for a charged cylindrical surface immersed in a 1-1 electrolyte can be written as

$$
\frac{\mathrm{d}^{2} \Psi}{\mathrm{d} r^{2}}+\frac{1}{r} \frac{\mathrm{d} \Psi}{\mathrm{d} r}=\kappa^{2} \sinh \Psi
$$

Here $r$ is the radial coordinate and $\Psi$ is the dimensionless potential. We consider the case of the double layer outside a cylinder. Making the coordinate transtormation $r=a+x / \kappa$ we find

$$
\begin{aligned}
& \frac{\mathrm{d}^{2}}{\mathrm{~d} x^{2}}\left[\Psi^{(0)}+\frac{1}{\kappa a} \Psi^{(1)}+\ldots\right]+\frac{1 / \kappa a}{1+x / \kappa a} \frac{\mathrm{d}}{\mathrm{d} x}\left[\Psi^{(0)}+\frac{1}{\kappa a} \Psi^{(1)}+\ldots\right] \\
& \quad=\sinh \left[\Psi^{(0)}+\frac{1}{\kappa a} \Psi^{(i)}+\ldots\right] .
\end{aligned}
$$

Along the same lines as for the case of the spherical surface we now find the following equations:

$$
\begin{aligned}
& \frac{\mathrm{d}^{2} \Psi^{(1)}}{\mathrm{d} x^{2}}=\sinh \Psi^{(1)}, \\
& \frac{\mathrm{d}^{2} \Psi^{(1)}}{\mathrm{d} x^{2}}-\Psi^{(1)} \cosh \left(\Psi^{(1)}\right)=-\frac{\mathrm{d}}{\mathrm{d} x} \Psi^{(1)},
\end{aligned}
$$




$$
\frac{\mathrm{d}^{2} \Psi^{(2)}}{\mathrm{d} x^{2}}-\Psi^{(2)} \cosh \left(\Psi^{(0)}\right)=-\frac{\mathrm{d}}{\mathrm{d} x} \Psi^{(1)}+x \frac{\mathrm{d}}{\mathrm{d} x} \Psi^{(0)}+\frac{1}{2}\left(\Psi^{(1)}\right)^{2} \sinh \Psi^{(0)}
$$

With the boundary condition (11) we now obtain

$$
\begin{aligned}
\frac{\mathrm{d} \Psi^{(0)}}{\mathrm{d} x}=-2 \sinh \left(\frac{1}{2} \Psi^{(0)}\right) & \\
\frac{\mathrm{d} \Psi^{(1)}}{\mathrm{d} x}+\Psi^{(1)} \cosh \left(\frac{1}{2} \Psi^{(0)}\right)= & -2 \tanh \left(\frac{1}{4} \Psi^{(0)}\right) \\
\frac{\mathrm{d} \Psi^{(2)}}{\mathrm{d} x}+\Psi^{(2)} \cosh \left(\frac{1}{2} \Psi^{(0)}\right)= & -\frac{1}{4}\left(\Psi^{(1)}\right)^{2} \sinh \left(\frac{1}{2} \Psi^{(0)}\right)-\frac{\Psi^{(1)}}{\cosh \left(\frac{1}{2} \Psi^{(0)}\right)+1} \\
& +2 x \tanh \left(\frac{1}{4} \Psi^{(0)}\right)+\frac{\tanh ^{2}\left(\frac{1}{4} \Psi^{(0)}\right)}{\sinh \left(\frac{1}{2} \Psi^{(0)}\right)}
\end{aligned}
$$

Using the boundary condition (16) one obtains from eqs. (26)-(28) to order $1 /(\kappa a)^{2}$ the following expression for the surface potential:

$$
\Psi_{0}=2 \ln (p+q)-\frac{1}{\kappa a} \frac{2(q-1)}{p q}+\frac{1}{(\kappa a)^{2}} \frac{(q-1)^{2}(2 q+1)}{p^{3} q^{3}},
$$

where $p$ and $q$ are given by eq. (18). For the electrical free energy we now obtain

$$
\begin{aligned}
f_{\mathrm{el}}^{\mathrm{cl} 1}= & \left(\frac{k_{\mathrm{B}} T}{e}\right)^{2} \varepsilon_{0} \varepsilon_{\mathrm{r}} \kappa\left\{4(p \ln (p+q)-q+1)-\frac{4}{\kappa a} \ln \left(\frac{1}{2}[q+1]\right)\right. \\
& \left.+\frac{1}{(\kappa a)^{2}} \frac{(q-1)(q+2)}{(q+1) q}\right\} .
\end{aligned}
$$

\section{Bending elastic moduii}

The curvature elastic free energy per unit area of a fluid layer can be written as

$$
f_{\mathrm{c}}=\frac{1}{2} K\left(c_{1}+c_{2}-c_{0}\right)^{2}+\bar{K} c_{1} c_{2},
$$

where $c_{1}$ and $c_{2}$ are the prircipal curvatures, $c_{0}$ is the spontaneous curvature, $K$ is the bending elastic modulus and $\bar{K}$ is the modulus of Gaussian curvature. For a sphere $c_{1}=c_{2}=1 / a$ and ihus eq. (31) takes the form 


$$
f_{c}^{\text {sphere }}=\frac{1}{2} K\left(\frac{2}{a}-c_{0}\right)^{2}+\bar{K}\left(\frac{1}{a}\right)^{2}
$$

For a cylinder $c_{1}=1 / a$ and $c_{2}=0$ and then the curvature elastic free energy takes the form

$$
f_{\mathrm{c}}^{\mathrm{cyl}}=\frac{1}{2} K\left(\frac{1}{a}-c_{0}\right)^{2} .
$$

We now compare the phenomenological expressions (32) and (33) with the expressions for the electrical free energy of a charged spherical and cylindrical surface (eqs. (20) and (30)). Comparing the coefficients of the $(1 / a)^{2}$ terms in the free energy expressions we obtain the following results for the contribution of the electric double layer to the elastic bending moduli of a charged monolayer:

$$
K^{\mathrm{cl}}=\frac{1}{2 \pi} \frac{k_{\mathrm{B}} T}{Q \kappa} \frac{(q-1)(q+2)}{(q+1) q}
$$

and

$$
\bar{K}^{\mathrm{el}}=\frac{-1}{\pi} \frac{k_{\mathrm{B}} T}{Q \kappa} \int_{z=2 /(1+q)}^{z=1} \frac{\ln z}{(z-1)} \mathrm{d} z .
$$

From the above expressions we see that the contribution of the electric double layer to the elastic bending moduli depends on the dimensionless quantities $Q_{\kappa}$ and $q$. These quantities in turn are determined by the salt concentration $n_{\mathrm{el}}$ and the surface charge $\sigma$. In order to get some feeling for the values of $K^{\mathrm{el}}$ and $\bar{K}^{\mathrm{el}}$, we consider as an example an amphiphilic layer encountered in microemulsions, namely a mixed monolayer of sodium dodecyl sulfate (SDS) and pentanol at the cyclohexarie/water interface [32]. In this mixed monolayer the adsorption of SDS is about $1.6 \mu \mathrm{mol} \mathrm{m}^{-2}$. Assuming complete ionisation of the SDS this leads to a surface charge of $0.154 \mathrm{C} \mathrm{m}^{-2}$. In table I we present values for $K^{\mathrm{el}}$ and $\bar{K}^{\mathrm{el}}$ in units $k_{\mathrm{B}} T$ for a surface charge of $0.15 \mathrm{C} \mathrm{m}^{-2}$ and salt concentrations of $0.01 \mathrm{M}$ to $0.40 \mathrm{M}$. We find that for these values of the parameters the electric contribution to the bending elastic moduli increases from about $0.1 k_{\mathrm{B}} T$ to $1 k_{\mathrm{B}} T$ with decreasing salt concentration. This trend is in gcod agreement with the experimental results [16] for $K$.

For small surface charge densities $p \ll i$ and thus $q \cong 1+\frac{1}{2} p^{2}$. We then find from eqs. (34) and (35) the following expressions for the elastic bending moduli of a charged monolayer: 
Table I

$K^{\text {el }}$ and $\bar{K}^{\text {el }}$ for a surface charge $|\sigma|=0.15 \mathrm{Cm}^{-2}$ and different $1-1$ electrolyte concentrations.

\begin{tabular}{lrrrrrr}
\hline & \multicolumn{6}{c}{ Electrolyte concentration (M) } \\
\hline & 0.01 & 0.02 & 0.05 & 0.10 & 0.20 & 0.40 \\
\hline$\kappa^{-1}(\mathrm{~nm})$ & 3.04 & 2.15 & 1.36 & 0.96 & 0.68 & 0.48 \\
& 12.8 & 9.12 & 5.82 & 4.17 & 3.03 & 2.25 \\
$K^{\text {el }} / k_{\mathrm{B}} T$ & 0.67 & 0.47 & 0.29 & 0.19 & 0.13 & 0.08 \\
$\bar{K}^{\text {el }} / k_{\mathrm{B}} T$ & -1.62 & -1.03 & -0.55 & -0.32 & -0.18 & -0.09 \\
\hline
\end{tabular}

$$
K^{\mathrm{el}}=\frac{k_{\mathrm{B}} T 3 \pi Q|\sigma|^{2}}{2 \kappa^{3} e^{2}}
$$

and

$$
\bar{K}^{\mathrm{el}}=-\frac{k_{\mathrm{B}} T \pi Q|\sigma|^{2}}{\kappa^{3} e^{2}} .
$$

These expressions are in agreement with the results obtained by Winterhalter and Helfrich [23] using the linearized Poisson-Boltzmann equation.

For high surface charge densities and low salt concentrations $p \cong q \gg 1$ and we now find the following expressions for the bending elastic moduli:

$$
\begin{aligned}
K^{\mathrm{el}} & =\frac{1}{2 \pi} \frac{k_{\mathrm{B}} T}{Q_{\kappa}}, \\
\bar{K}^{\mathrm{el}} & =-\frac{\pi}{6} \frac{k_{\mathrm{B}} T}{Q_{\kappa}} .
\end{aligned}
$$

Under these conditions $\left(2 K^{\mathrm{el}}+\bar{K}^{\mathrm{el}}\right)<0$ which might have important implications for spontaneous vesiculation as discussed by Helfrich and coworkers $[6,23]$. We note that even in the case of high surface charge densities only for rather low salt concentrations (below $10^{-2} \mathrm{M}$ ) the electric contribution to the bending elastic modulus will exceed $k_{\mathrm{B}} T$. Therefore it is not unreasonable to surmise as did Szleifer et al. [20] that in general the curvature elasticity of surfactant films is dominated by the "tails" - rather than by the "heads" - of the constituent amphiphiles. Nevertheless in the case of monolayers encountered in microemulsion systems, where the curvature elasticity is low (of the order of $k_{\mathrm{B}} T$ ) the electric contribution is significant and has to be taken into account. 


\section{Acknowledgements}

The author is grateful to Prof. J.Th.G. Overbeek and Prof. Th. Odijk for extensive discussions on electric double layer theory.

\section{References}

[1] W. Helfrich, Z. Naturforsch. 28C (1973) 693.

[2] A.G. Petrov and I. Bivas, Progr. Surf. Sci. 16 (1984) 389.

[3] Physics of Amphiphilic Layers, J. Meunier, D. Langevin and N. Boccara, ed. (Springer, Berlin, 1987).

[4] P.G. de Gennes and C. Taupin, J. Phys. Chem. 86 (1982) 2294.

[5] D. Andelman, M.E. Cates, D. Roux and S.A. Safran, J. Chem. Phys. 87 (1987) 7229.

[6] W. Helfrich and W. Harbich, ref. [3], p. 58.

[7] R.M. Servuss, W. Harbich and W. Helfrich, Biochim. Biophys. Acta 436 (1976) 900.

[8] M.B. Schneider, J.T. Jenkins and W.W. Webb, J. Phys. (Paris) 45 (1984) 1457.

[9] H. Engelhardt, H.P. Duwe and E. Sackmann, J. Phys. (Paris) 46 (1985) L395.

[10] G. Beblik, R.M. Servuss and W. Helfrich, J. Phys. (Paris) 46 (1985) 1773.

[11] I. Bivas, P. Hanusse, P. Bothorel, J. Lalanne and O. Agueree-Chariol, J. Phys. (Paris) 48 (1987) 855.

[12] E. Evans, Biophys. J. 43 (1983) 27.

[13] I.M. Di Meglio, M. Dvolaitzky and C. Taupin, J. Phys. Chem. 89 (1985) 871.

[14] J.M. Di Meglio, M. Dvolaitzky, L. Légor and C. Taupin, Phys. Rev. Lett. 54 (1985) 1686.

[15] F. Nallet, D. Roux and J. Prost, Phys. Rev. Lett. 62 (1989) 276.

[16] J. Meunier, J. Phys. (Paris) 46 (1985) L1005.

[17] J. Meunier, J. Phys. (Paris) 48 (1987) 1819.

[18] C.R. Safinya, D. Roux, G.S. Smith, S.K. Sinha, P. Dimon, N.A. Clark and A.M. Bellocq, Phys. Rev. Lett. 57 (1986) 2718.

[19] D. Roux and C.R. Safinya, J. Phys. (Paris) 49 (1988) 307.

[20] I. Szleifer, D. Kramer, A. Ben-Shaul, D. Roux and W.M. Gelbart. Phys. Rev, Lett. 60 (1988) 1966.

[21] J.Th.G. Overbeek, Faraday Discuss. Chem. Soc. 65 (1978) 7.

[22] J.Th.G. Overbeek, G.J. Verhoeckx, P.L. De Bruyn and H.N.W. Lekkerkerker, J. Colloid Interface Sci. 119 (1987) 422.

[23] N. Winterhalter and W. Helfrich, J. Phys. Chem. 92 (1988) 6865.

[24] T. Odijk, J. Polym. Sci., Polym. Phys. Ed. 15 (1977) 477.

[25] J. Skolnick and M. Fixman, Macromolecules 10 (1977) 944.

[26] M. Le Bret, J. Chem. Phys. 76 (1982) 6243.

[27] M. Fixman, J. Chem. Phys. 76 (1982) 6346.

[28] E.J.W. Verwey and J.Th.G. Overbeek, Theory of the Stability of Lyophobic Colloids (Elsevier, Amsterdam, 1948), p. 53.

[29] S.S. Dukhin, N.M. Semenikhin and L.M. Shapinskaya, Dokl. Phys. Chem. 193 (1970) 540.

[30] W.C. Chen and P.N. Sen, J. Chem. Phys. 77 (1982) 2042.

[31] A.N. Stokes, J. Chem. Phys. 65 (1976) 261.

[32] G.J. Verhoeckx. P.L. De Bruyn and J.Th.G. Overbeek, J. Colloid Interface Sci. 119 (1987) 409. 